ARTICLE

DOI: $10.1038 / s 41467-017-01177-0$

\title{
Breakdown of magnons in a strongly spin-orbital coupled magnet
}

\author{
Stephen M. Winter (1D ${ }^{1}$, Kira Riedl ${ }^{1}$, Pavel A. Maksimov², Alexander L. Chernyshev², Andreas Honecker (D) ${ }^{3}$ \\ \& Roser Valentí
}

The description of quantized collective excitations stands as a landmark in the quantum theory of condensed matter. A prominent example occurs in conventional magnets, which support bosonic magnons-quantized harmonic fluctuations of the ordered spins. In striking contrast is the recent discovery that strongly spin-orbital-coupled magnets, such as $\alpha-\mathrm{RuCl}_{3}$, may display a broad excitation continuum inconsistent with conventional magnons. Due to incomplete knowledge of the underlying interactions unraveling the nature of this continuum remains challenging. The most discussed explanation refers to a coherent continuum of fractional excitations analogous to the celebrated Kitaev spin liquid. Here, we present a more general scenario. We propose that the observed continuum represents incoherent excitations originating from strong magnetic anharmonicity that naturally occurs in such materials. This scenario fully explains the observed inelastic magnetic response of $\alpha-\mathrm{RuCl}_{3}$ and reveals the presence of nontrivial excitations in such materials extending well beyond the Kitaev state.

\footnotetext{
${ }^{1}$ Institut für Theoretische Physik, Goethe-Universität Frankfurt, Max-von-Laue-Strasse 1, 60438 Frankfurt am Main, Germany. ${ }^{2}$ Department of Physics and Astronomy, University of California, Irvine, CA 92697, USA. ${ }^{3}$ Laboratoire de Physique Théorique et Modélisation, CNRS UMR 8089, Université de CergyPontoise, 95302 Cergy-Pontoise Cedex, France. Correspondence and requests for materials should be addressed to S.M.W. (email: winter@physik.uni-frankfurt.de)
} 
$\mathrm{F}$ rom magnons in ordered magnets to phonons in periodic crystals, the appearance of bosonic collective excitations is ubiquitous in condensed phases of matter ${ }^{1}$. For this reason, special attention is given to those states that support more exotic collective modes, for which the conventional paradigm breaks down. In the context of magnetic phases, the breakdown of magnons is commonly thought to require closeness to an unconventional state such as a quantum spin liquid ${ }^{2-4}$. A notable example occurs in Kitaev's exactly solvable honeycomb model ${ }^{5}$, for which strongly anisotropic and bond-dependent interactions fractionalize conventional spin excitations into Majorana spinons and fluxes. This Kitaev state has recently risen to prominence due to the suggestion that it may be realized in heavy metal $4 d^{5}$ and $5 d^{5}$ insulators via a specific interplay between the crystal field and strong spin-orbit coupling ${ }^{6}$, and, consequently, a variety of candidate materials based on $\mathrm{Ir}^{4+}$ and $\mathrm{Ru}^{3+}$ have been intensively explored ${ }^{7}$. Encouragingly, evidence of a continuum of magnetic excitations that is inconsistent with conventional magnons was found in the majority of such materials, including the twodimensional (2D) honeycomb $\mathrm{Na}_{2} \mathrm{IrO}_{3}{ }^{8,9}$ and $\alpha-\mathrm{RuCl}_{3}{ }^{10-14}$, as well as the three-dimensional (3D) analogs $\beta-, \gamma-\mathrm{Li}_{2} \mathrm{IrO}_{3}{ }^{15}$, despite all of them having magnetically ordered ground states.

While the observed excitation continua in these systems have been interpreted in terms of signatures of the Kitaev state, the low-symmetry crystalline environment of the real materials also allows various additional interactions beyond Kitaev's model ${ }^{16-}$ 18 , which are thought to be large based on both experimental ${ }^{19,20}$ and theoretical ${ }^{18}, 21,22$ considerations. In this sense, understanding the mechanism for the breakdown of magnons and the appearance of a broad continuum of magnetic excitations remain a key challenge.

In this work, we study a representative case $\alpha-\mathrm{RuCl}_{3}$, which forms a layered $2 \mathrm{D}$ honeycomb lattice and displays zigzag magnetic order below $T_{\mathrm{N}} \sim 7 \mathrm{~K}^{12}, 13,23$. We specifically address the recent inelastic neutron scattering (INS) measurements, which have revealed low-energy magnons ${ }^{24}$ coexisting with an intense excitation continuum ${ }^{12}$. The latter continuum possesses a distinctive six-fold star shape in momentum space, and large intensity at the $2 \mathrm{D} \Gamma$-point over a wide energy range $E=2-15 \mathrm{meV}^{12}$. To resolve the nature of this continuum, we take two complementary approaches. We first theoretically investigate the neutron spectra over a range of relevant magnetic interactions in order to determine the correct spin Hamiltonian for $\alpha-\mathrm{RuCl}_{3}$, which has been a subject of intense recent discussion ${ }^{18,25-28}$. Second, we identify the conditions that lead to the breakdown of conventional magnons in the presence of strongly anisotropic and frustrated interactions, revealing that nontrivial excitations naturally persist well beyond the Kitaev spin liquid.

\section{Results}

The model. Based on previous ab initio studies ${ }^{18,25-28}$, the largest terms in the spin Hamiltonian of $\alpha-\mathrm{RuCl}_{3}$ are generally expected to include nearest neighbor Heisenberg $J_{1}$, Kitaev $K_{1}$, and off-diagonal $\Gamma_{1}$ couplings, supplemented by a third neighbor Heisenberg $J_{3}$ term:

$$
\begin{aligned}
\mathcal{H}= & \sum_{\langle i, j\rangle} J_{1} \mathbf{S}_{i} \cdot \mathbf{S}_{j}+K_{1} S_{i}^{\gamma} S_{j}^{\gamma}+\Gamma_{1}\left(S_{i}^{\alpha} S_{j}^{\beta}+S_{i}^{\beta} S_{j}^{\alpha}\right) \\
& +\sum_{\langle\langle\langle i, j\rangle\rangle\rangle} J_{3} \mathbf{S}_{i} \cdot \mathbf{S}_{j}
\end{aligned},
$$

where $\langle i, j\rangle$ and $\langle\langle\langle i, j\rangle\rangle\rangle$ refer to summation over first and third neighbor bonds, respectively (see Fig. 1). The bond-dependent variables $\{\alpha, \beta, \gamma\}$ distinguish the three types of first neighbor bonds, with $\{\alpha, \beta, \gamma\}=\{y, z, x\},\{z, x, y\}$, and $\{x, y, z\}$ for the Xbonds, Y-bonds, and Z-bonds, respectively. The third neighbor

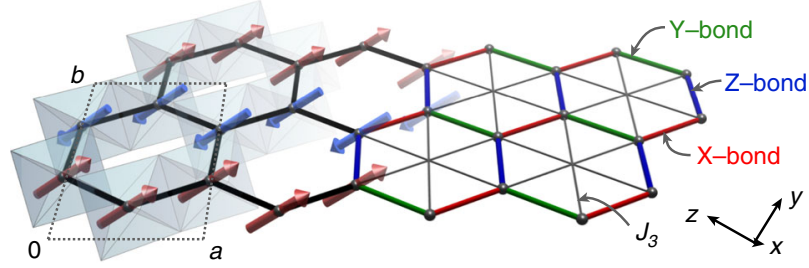

Fig. 1 From material to model. Within the honeycomb ab-layer of $\alpha-\mathrm{RuCl}_{3}$ are illustrated the $\mathrm{RuCl}_{6}$ octahedra, magnetic zigzag ordering pattern, and definition of the underlying magnetic interactions. Crystal axes are labeled with respect to the $\mathrm{C} 2 / \mathrm{m}$ structure

interactions are bond-independent. The phase diagram of this model has been discussed previously $17,18,26,29$, and is further detailed in Supplementary Note 1; here we review the key aspects.

In the limit $J_{1}=\Gamma_{1}=J_{3}=0$, the ground state is a gapless $Z_{2}$ spin liquid for either positive or negative $K_{1}$, as demonstrated in Kitaev's seminal work ${ }^{5}$. Small perturbations from the pure $K_{1}$ limit may induce various magnetically ordered states, such as the zigzag antiferromagnetic (AFM) state observed in $\alpha-\mathrm{RuCl}_{3}$ and shown in Fig. 1. The simplest perturbation is the introduction of a finite $J_{1}$, which yields the well-studied $\left(J_{1}, K_{1}\right)$ nearest neighbor Heisenberg-Kitaev (nnHK) model. This model hosts zigzag order in the region $K_{1}>0, J_{1}<0$, as discussed in Supplementary Note 1. Accordingly, previous analysis of the powder INS experiments within the context of the nnHK model ${ }^{\mathrm{f}}{ }^{\text {suggested that } K_{1} \sim+7}$ $\mathrm{meV}$, and $\left|J_{1} / K_{1}\right| \sim 0.3-0.7$ for $\alpha-\mathrm{RuCl}_{3}$. On this basis, the excitation continua observed experimentally were initially interpreted in terms of proximity to the AFM $K_{1}>0$ spin liquid ${ }^{12,13}$. However, the further consideration of finite $\Gamma_{1}$ and $J_{3}$ interactions in Eq. (1) significantly expands the experimentally relevant region, as both interactions generally stabilize zigzag order. Indeed, recent ab initio studies ${ }^{18,25-28}$ have suggested that the zigzag order in $\alpha-\mathrm{RuCl}_{3}$ emerges from $J_{1} \sim 0, K_{1}<0, \Gamma_{1}>0$, and $J_{3}>0$, with $\left|\Gamma_{1} / K_{1}\right| \sim 0.5-1.0$ and $\left|J_{3} / K_{1}\right| \sim 0.1-0.5$, as reviewed in Supplementary Note 2 . That is, $K_{1}$ is ferromagnetic, and supplemented by significant $\Gamma_{1}$ and $J_{3}$ interactions. Such interactions would represent large deviations from both Kitaev's original model and the region identified by initial experimental analysis. Before discussing the origin of the excitation continua, it is therefore crucial to first pinpoint the relevant interaction parameters.

In order to address this issue directly, we have computed the neutron scattering intensity $\mathcal{I}(\mathbf{k}, \omega)$ for a variety of interactions within the zigzag ordered phase via both linear spin-wave theory (LSWT) and exact diagonalization (ED). For the latter case, we combine results from various periodic 20 -site and 24-site clusters compatible with the zigzag state in order to probe a wider range of k-points (see "Methods" section). Full results for the complete range of models are presented in Supplementary Note 5. Here, we highlight the key results for two representative sets of interactions. Within the $\left(J_{1}, K_{1}\right)$ nnHK model, we focus on Model $1\left(J_{1}\right.$ $\left.=-2.2, K_{1}=+7.4 \mathrm{meV} ;\left|J_{1} / K_{1}\right|=0.3\right)$, which lies on the border of the region initially identified in ref. ${ }^{12}$, close to the spin liquid. Beyond the nnHK model, we consider Model $2\left(J_{1}=-0.5, K_{1}=\right.$ $-5.0, \Gamma_{1}=+2.5, J_{3}=+0.5 \mathrm{meV}$ ) for which parameters have been guided by recent ab initio studies ${ }^{18,25-28}$, and further optimized to improve agreement with the experimental spectra. Results for Models 1 and 2 are first presented in Figs. 2 and 3, which show detailed $\omega$-dependence and $\mathbf{k}$-dependence of $\mathcal{I}(\mathbf{k}, \omega)$, along with the evolution of the spectra upon changing parameters toward the $K_{1}>0$ or $K_{1}<0$ spin liquid regions.

Nearest neighbor Heisenberg-Kitaev model. We begin by analyzing the spectra $\mathcal{I}(\mathbf{k}, \omega)$ within the zigzag phase of the $\left(J_{1}, K_{1}\right)$ 


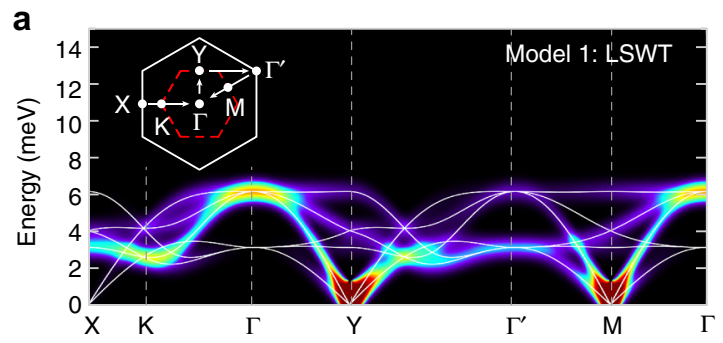

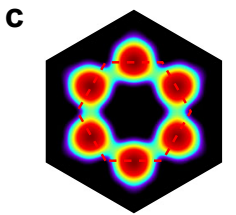

$E=[1.3,2.3] \mathrm{meV}$

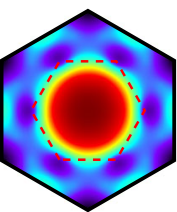

$[6.0,9.0]$

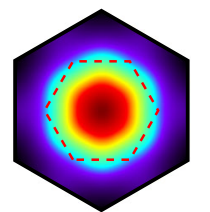

$[10.5,20.0]$

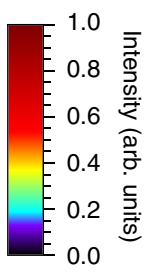

e

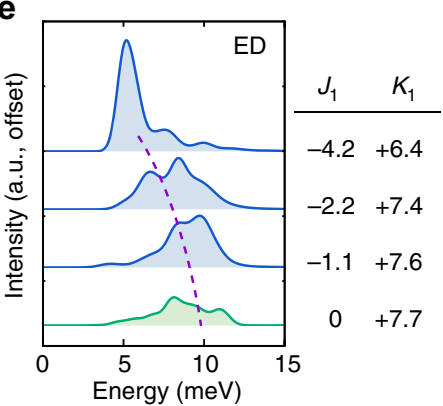

.

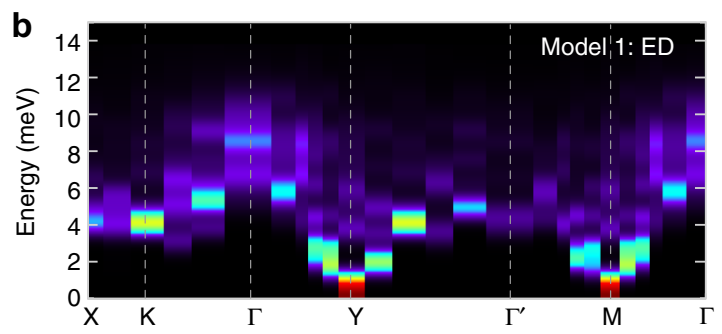

d

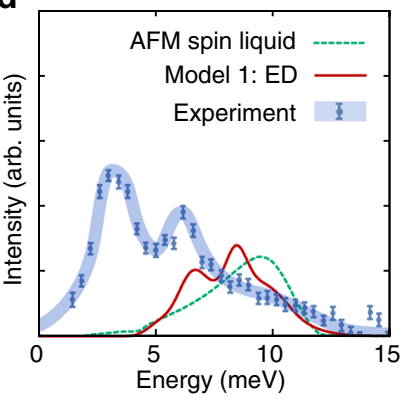

(1) 


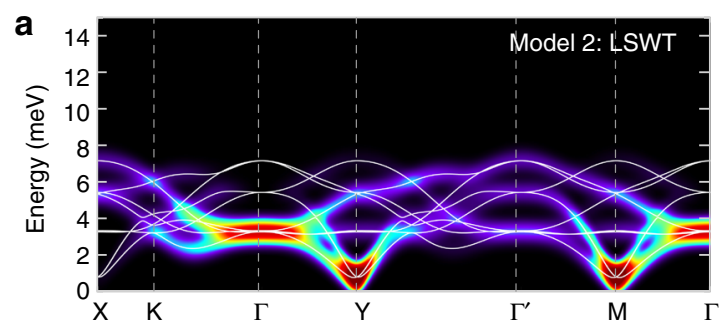

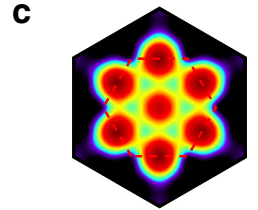

$E=[1.3,2.3] \mathrm{meV}$

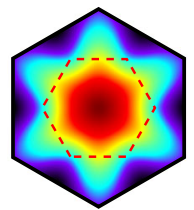

$[5.5,8.5]$

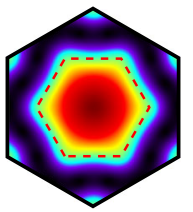

$[10.5,20.0]$

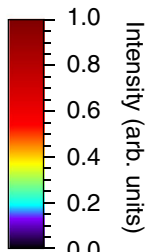

0.0
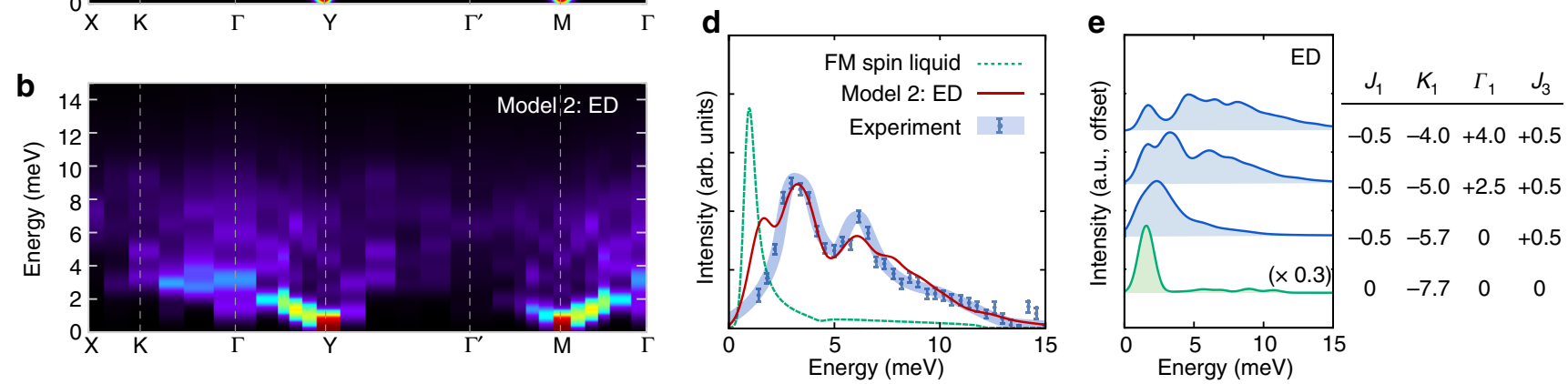

Fig. 3 Neutron scattering intensity $\mathcal{I}(\mathbf{k}, \omega)$ within the extended model. a-c Detailed results for Model $2\left(J_{1}=-0.5, K_{1}=-5.0, \Gamma_{1}=+2.5, J_{3}=+0.5\right.$ meV): a $\mathcal{I}(\mathbf{k}, \omega)$ computed via LSWT; results are averaged over the three zigzag domains with ordering wavectors parallel to the $\mathrm{X}$-bonds, $\mathrm{Y}$-bonds, and Z-bonds. $\mathbf{b}$ ED results, combining data from several 20-site and 24-site periodic clusters (see "Methods"). c ED $\mathbf{k}$-dependence of $\mathcal{I}(\mathbf{k}, \omega)$ integrated over the indicated energies, as obtained from a single 24-site cluster respecting all symmetries of Eq. (1) (see "Methods"). $\mathbf{d}$ Comparison of $\Gamma$-point intensities for the $K_{1}=$ -7.7 meV FM spin liquid (exact results ${ }^{53,54}$ ), Model 2 (ED), and the experimental data for $\alpha$ - $\mathrm{RuCl}_{3}{ }^{12}$. e Evolution of the ED $\Gamma$-point intensity with decreasing $\left|\Gamma_{1} / K_{1}\right|$, showing significant broadening at finite $\Gamma_{1}$. The top three interaction sets correspond to zigzag order, while the bottom is the $K_{1}<0$ spin liquid. For all spectra, a Gaussian broadening of $0.5 \mathrm{meV}$ has been applied

fluctuations associated with the higher-order anharmonic decay terms may mix sharp single-magnon modes with the multimagnon continuum ${ }^{32-34}$. Similar considerations also apply to the breakdown of other collective modes, such as phonons in anharmonic crystals ${ }^{35,36}$. From this perspective, a large decay rate is expected for any single-magnon mode that is energetically degenerate with the multi-particle continuum, unless there are specific symmetries guaranteeing that the two do not couple. It is therefore useful to consider the prerequisites for magnon breakdown in the presence of the strongly anisotropic interactions of Eq. (1).

Magnon decay channels for the nnHK model. We first examine the stability of magnons in the nnHK model. For pure $J_{1}$ and $K_{1}$ interactions, the total spin projections $S_{\text {tot }}^{\gamma}=\sum_{i} S_{i}^{\gamma}$ are conserved along the cubic axes $\gamma=\{x, y, z\}$ modulo two. Since the ordered moment also lies along one of the cubic axes in the zigzag phase $^{20,37}$ (see Fig. 4c), the possible magnon decay channels are restricted. In the local picture, the relevant quantum fluctuations are local singlet $S_{i}^{x} S_{j}^{x}|\uparrow \downarrow\rangle=|\downarrow \uparrow\rangle$ and triplet $S_{i}^{x} S_{j}^{x}|\uparrow \uparrow\rangle=|\downarrow \downarrow\rangle$ fluctuations shown in Fig. $4 \mathrm{a}$, with $\Delta S_{\text {tot }}^{z}=0$ and 2, respectively. In the magnon picture, the Hamiltonian can only contain evenorder terms (i.e., $\mathcal{H}=\mathcal{H}_{2}+\mathcal{H}_{4}+\ldots$ ), analogous to conventional Heisenberg antiferromagnets with collinear ordered spins ${ }^{32,34}$. For example, the fourth-order decay process due to $\mathcal{H}_{4}$ mixes the one-magnon states with the three-magnon continuum $\left(\Delta N_{\text {tot }}=\right.$ \pm 2 ), where

$$
\mathcal{H}_{4}=\sum_{1-4} V_{123}^{4} a_{1}^{\dagger} a_{2}^{\dagger} a_{3}^{\dagger} a_{4}^{\dagger} \delta\left(\mathbf{k}_{1}+\mathbf{k}_{2}+\mathbf{k}_{3}-\mathbf{k}_{4}\right)+\text { H.c. }
$$

Here, the bold index $\left(\mathbf{n} \equiv \mathbf{k}_{n}, m_{n}\right)$ labels both momentum and magnon band. This process is pictured in Fig. 4b. As noted above, the effect of such terms depends crucially on the availability of low-energy three-magnon states in which to decay.

The density of three-magnon states for Model 1 is shown in Fig. 4 d, based on the one-magnon dispersions obtained in LSWT. At each k-point, the lowest energy three-magnon state

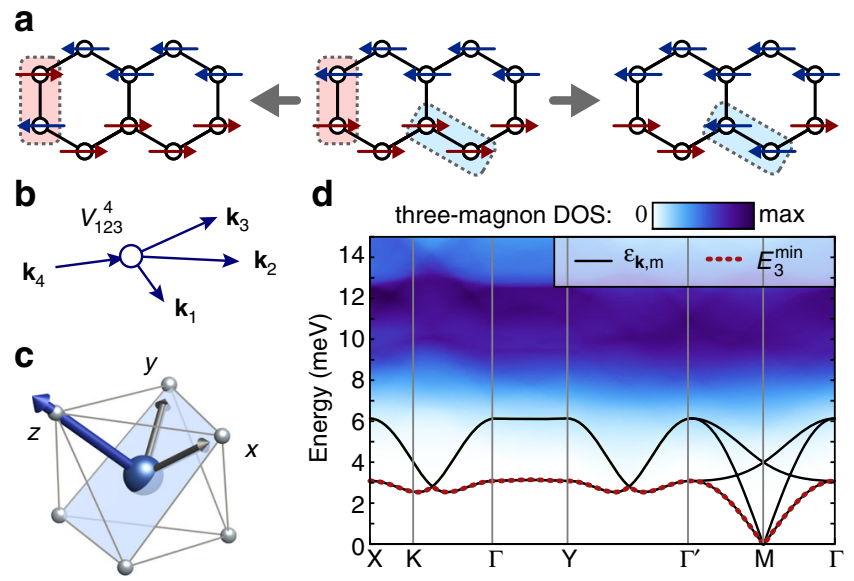

Fig. 4 Magnon decay channels for the nnHK model. a Local picture of quantum fluctuations away from zigzag order. The energy cost for the left process vanishes on approaching the spin liquid $\left|J_{1} / K_{1}\right| \rightarrow 0$. b Momentum space picture for the corresponding fourth-order decay process due to $\mathcal{H}_{4}$ c Ordered moment direction for Model $1\left(J_{1}=-2.2, K_{1}=+7.4 \mathrm{meV}\right)$, corresponding to the zigzag domain with ordering wavevector $\mathbf{Q}=\mathrm{Y}$. d LSWT dispersions $\epsilon_{\mathbf{k}, m}$, and three-magnon density of states (DOS) for Model 1 for the same zigzag domain as $\mathbf{c}$. The dashed line indicates the bottom of the three-magnon continuum $\left(E_{3}^{\min }\right)$, which is coincident with the lowest magnon band

$a_{\mathbf{q}_{1}}^{\dagger} a_{\mathbf{q}_{2}}^{\dagger} a_{\mathbf{q}_{3}}^{\dagger}|0\rangle$ (with $\mathbf{q}_{1}+\mathbf{q}_{2}+\mathbf{q}_{3}=\mathbf{k}$ ) is obtained by placing two particles in the pseudo-Goldstone modes at opposite M-points $\left(\mathbf{q}_{1}+\mathbf{q}_{2}=0\right)$, and the third particle at $\mathbf{q}_{3}=\mathbf{k}$, with total energy $E_{3}^{\min }(\mathbf{k})=\epsilon_{\mathbf{k}, 1}+2 \epsilon_{\mathrm{M}, 1}$. This implies $E_{3}^{\min }(\mathbf{k}) \geq \epsilon_{\mathbf{k}, 1}$. That is, the three-magnon states lie above the lowest one-magnon band at every k-point. As a result, every magnon in the lowest band remains kinetically stable, due to the absence of low-energy threeparticle states in which to decay. Precisely this condition ensures the stability of low-energy magnons in conventional isotropic antiferromagnets, and explains the sharp magnon-like peaks 


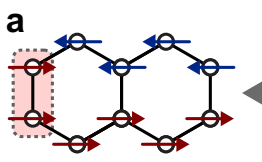

\section{4}

b
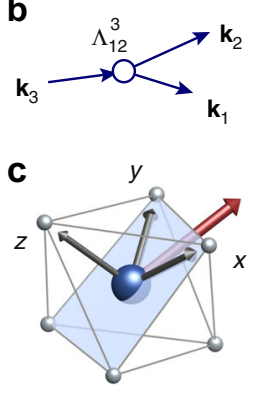

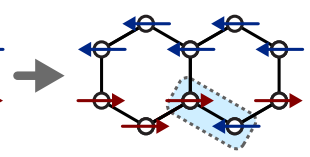

d

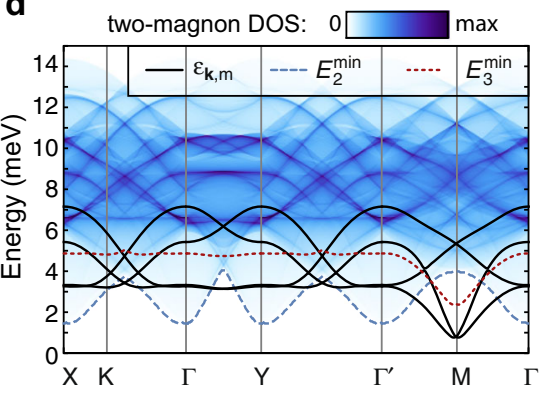

Fig. 5 Magnon decay channels for the extended model. a Local picture of additional quantum fluctuations away from zigzag order induced by $\Gamma_{1}$ interactions. b Momentum space picture of the third-order decay process $\mathcal{H}_{3}$. c Ordered moment direction for Model $2\left(J_{1}=-0.5, K_{1}=-5.0, \Gamma_{1}=\right.$ $+2.5, J_{3}=+0.5 \mathrm{meV}$ ) with zigzag ordering wavevector $\mathbf{Q}=\mathrm{Y}$, parallel to the Z-bond. d LSWT dispersions $\epsilon_{\mathbf{k}, m}$, and two-magnon DOS for Model 2 with $\mathbf{Q}=\mathrm{Y}$. Dashed lines indicate the bottom of the two-magnon and threemagnon continuum $\left(E_{2}^{\min }(\mathbf{k})\right.$ and $E_{3}^{\min }(\mathbf{k})$, respectively)

observed in Fig. 2b for Model 1. Strong spectral broadening in the nnHK model can occur only for high-lying excitations with $\epsilon_{\mathbf{k}, m}>\epsilon_{\mathbf{k}, 1}=E_{3}^{\mathrm{min}}$, where the density of three-magnon states is finite, such as at the $2 \mathrm{D} \Gamma$-point. On approaching the spin liquid (at $J_{1} / K_{1}=0$ ), this condition is relaxed due to the vanishing dispersion of the lowest magnon band (i.e., $\epsilon_{\mathbf{k}, 1} \rightarrow 0$ ), which corresponds to a vanishing energy cost the singlet fluctuations shown on the left of Fig. 4a. The relevant fluctuations in the limit $J_{1} / K_{1} \rightarrow 0$ therefore correspond to $\Delta N_{\text {tot }}= \pm 2$. For other values of $J_{1} / K_{1}$, the majority of magnons are expected to remain stable due to the absence of low-energy three-magnon states.

Magnon decay channels for the extended model. In Model 2, the character of the quantum fluctuations away from zigzag order is notably different (Fig. 5). The finite $\Gamma_{1}$ interaction reduces the local symmetry and leads to rotation of the ordered moments away from the cubic axes ${ }^{20,37}$ (Fig. 5c). In the local picture, this allows additional single-spin fluctuations $S_{i}^{x} S_{j}^{z}|\uparrow \uparrow\rangle=|\downarrow \uparrow\rangle$ (Fig. 5a), which correspond to odd-order anharmonic terms $\mathcal{H}_{3}, \mathcal{H}_{5}, \ldots$ in the magnon Hamiltonian, where ${ }^{33,34}$ :

$$
\mathcal{H}_{3}=\sum_{1-3} \Lambda_{12}^{3} a_{1}^{\dagger} a_{2}^{\dagger} a_{3} \delta\left(\mathbf{k}_{1}+\mathbf{k}_{2}-\mathbf{k}_{3}\right)+\text { H.c. }
$$

At lowest order, such terms mix the single-magnon states with the two-magnon continuum $\left(\Delta N_{\text {tot }}= \pm 1\right)$, via the scattering process depicted in Fig. 5b. The density of two-magnon states is shown in Fig. 5d, for the zigzag domain with $\mathbf{Q}=\mathrm{Y}$. In this case, at each k-point the lowest energy two-magnon state $a_{\mathbf{q}_{1}}^{\dagger} a_{\mathbf{q}_{2}}^{\dagger}|0\rangle$ is obtained by placing one particle in the pseudo-Goldstone mode at an $\mathrm{M}$-point, and the second particle at $\mathbf{q}_{2}=\mathbf{k}-\mathbf{M}$, with total energy $E_{2}^{\min }(\mathbf{k})=\epsilon_{\mathbf{k}-\mathrm{M}}+\epsilon_{\mathrm{M}} \neq E_{3}^{\min }$. It should be emphasized that this condition differs from that of a conventional Heisenberg antiferromagnet (for which $\left.E_{2}^{\min }=E_{3}^{\mathrm{min}}\right)^{34}$. In the case of Model 2 , the difference is directly related to the strong anisotropic $K_{1}$ and $\Gamma_{1}$ interactions, which shift the pseudo-Goldstone modes to the M-points, such that only high-energy magnons remain at the $\Gamma$-point or ordering wavevector $\mathbf{Q}^{38}$. This shift therefore leads to an offset of the low-energy even and odd magnon states in

k-space such that $E_{2}^{\min }(\mathbf{k})<\epsilon_{\mathbf{k}, 1}$ over a wide region of the Brillouin zone; there are many two-magnon states with equal or lower energy than the one-magnon states. Provided there is a finite $\Gamma_{1}$, the spontaneous decay of single magnons into the two-particle continuum is therefore allowed even for the lowest magnon band. The decay rate is expected to be particularly large near the zone center, which represents a minimum in $E_{2}^{\mathrm{min}}$. Similar kinematic conditions may also occur in other systems ${ }^{34,39}$. For Model 2, the pseudo-Goldstone magnons near the M-points remain coherent due to the absence of low-energy two particle states in which to decay (Fig. 5d). This explains the experimental observation of sharp magnon-like modes near the M-points ${ }^{24}$. In contrast, the magnon bands in the remainder of the Brillouin zone directly overlap with the two-particle continuum. It is therefore natural to anticipate a large decay rate even for the lowest magnon bands.

To confirm this idea, we have computed the three-magnon interactions and decay rates for all magnon bands for Model 2 using the self-consistent imaginary Dyson equation (iDE) approach $^{40}$. Within this approach, it is assumed that the real part of the magnon self-energy is already captured by the LSWT parameters, while the imaginary part is obtained self-consistently (see "Methods" and Supplementary Note 3). The iDE approach therefore represents an extension of LSWT, in which the onemagnon excitations are broadened according to the momentum and band-dependent decay rate $\gamma_{\mathbf{k}, n}$, while other contributions to the neutron intensity from multi-magnon excitations are also absent $^{41}$. As a result, comparison of LSWT, ED, and iDE results (Fig. 6) allows for the identification of the origin of different contributions to the spectra.

The predicted neutron scattering intensity within the iDE approach (Fig. 6b) captures many of the most notable features that are observed in the ED and experimental data, showing a significant improvement over the LSWT results (Fig. 6a). First, there is an almost complete washout of the two high-energy one-magnon modes due to strong decays. This implies that the higher-energy features $>4 \mathrm{meV}$ appearing in $\mathrm{ED}$ are primarily multi-magnon in character (including the $6 \mathrm{meV}$ peak at the $\Gamma$-point). The appearance of these higher-energy features in the inelastic neutron response may arise partly from direct contributions from the broadened two-magnon continuum via the longitudinal component of the structure factor, which is not included in the iDE approach (see Supplementary Note 3). Second, the broadening of the two lower magnon bands in the iDE results and the resultant variation of their intensities are in a close agreement with the ED-particularly in a wide region near the $\Gamma$-point (see also Supplementary Fig. 5). These are precisely the features with which the LSWT results were most incompatible. Over much of the Brillouin zone-and especially for the higher magnon bands - the computed $\gamma_{\mathbf{k}, n}$ is on the same scale as the one-magnon bandwidth, confirming the absence of coherent magnons.

\section{Discussion}

The general requirements for strong two-magnon decays are less restrictive than a proximity to a spin liquid state. Indeed, a large decay rate is ensured by the following three conditions: large anisotropic interactions, deviation of the ordered moments away from the high-symmetry axes, and strong overlap of the onemagnon states with the multi-magnon continuum (see Supplementary Note 3 ). Of these, the first two conditions ensure that the scattering vertex $\Lambda_{12}^{3}$ is large-of the order of the underlying interactions, i.e., $\Lambda_{12}^{3} \sim \mathcal{O}\left(K_{1}, \Gamma_{1}\right)$. For $\alpha-\mathrm{RuCl}_{3}$, the strong overlap with the multi-magnon continuum is ensured by shifting of the low-energy magnons away from the $\Gamma$-point. Since the bottom of the two-magnon continuum must always have an 

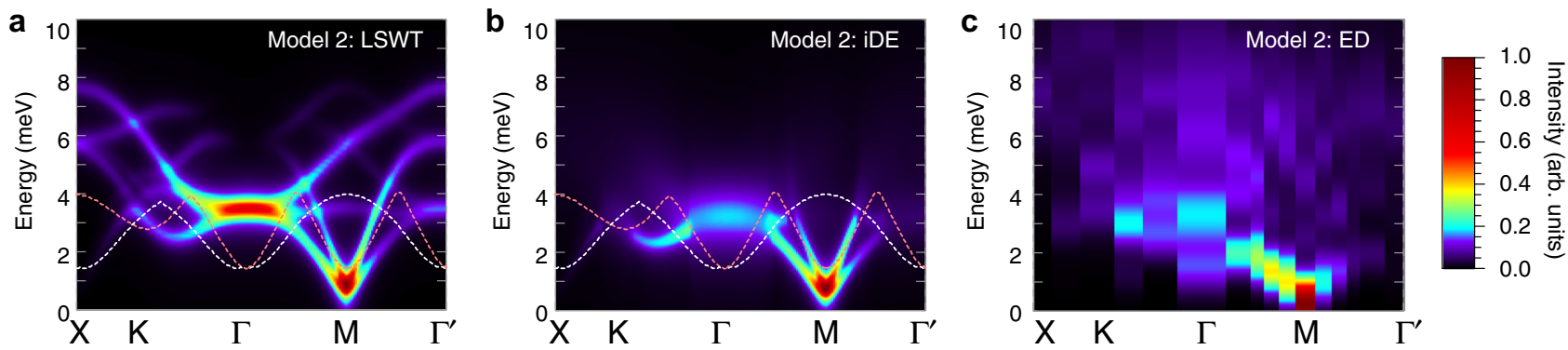

Fig. 6 Effects of two-magnon decays in $\mathcal{I}(\mathbf{k}, \omega)$ for extended model. Results are shown for Model 2 computed via a LSWT, b self-consistent iDE approach, and $\mathbf{c}$ ED. Results in $\mathbf{a}$ and $\mathbf{b}$ are averaged over the different zigzag domains. The white and pink dashed lines indicate the bottom of the two-magnon continuum, $E_{2}^{\min }(\mathbf{k})$ for the different zigzag domains. In the iDE results, the effects of two-magnon decays strongly broadens any magnon bands overlapping with the two-magnon continuum

energetic minimum at the $\Gamma$-point, the shifting of the pseudoGoldstone modes to a finite momentum ensures the remaining higher-energy magnons are degenerate with the continuum near the zone center. Experimentally, these conditions are also likely to be satisfied by the zigzag ordered $\mathrm{Na}_{2} \mathrm{IrO}_{3}{ }^{9}$, and spiral magnets $\alpha$ $\mathrm{Li}_{2} \mathrm{IrO}_{3}, \beta-\mathrm{Li}_{2} \mathrm{IrO}_{3}$, and $\gamma-\mathrm{Li}_{2} \mathrm{IrO}_{3}{ }^{42-44}$. This picture is also consistent with recent indications that the magnetically disordered phase observed at high pressure in $\beta-\mathrm{Li}_{2} \mathrm{IrO}_{3}{ }^{45}$ is driven primarily by large $\Gamma_{1}$ interactions ${ }^{46}$.

With this in mind, there are two general scenarios that can explain the observed continuum excitations in $\alpha-\mathrm{RuCl}_{3}$ and the iridates $A_{2} \mathrm{IrO}_{3}$. In the first scenario, which has been advanced by several studies, the excitations can be treated as free particles with a small number of flavors. Such excitations are weakly interacting and have well-defined dispersions, but possess quantum numbers (e.g., $\Delta S_{\text {tot }}= \pm 1 / 2$ ) or topological properties inconsistent with the experimental neutron scattering selection rules (i.e., $\Delta S_{\text {tot }}=0$, \pm 1 ). The appearance of the broad continuum in energy therefore results only from the fact that these fractional excitations must be created in multiples. If they could have been created individually, they would have represented long-lived and coherent quasiparticles with sharply peaked energies. This scenario indeed describes the Kitaev spin liquid, where the special symmetries of the Hamiltonian allow an exact description in terms of two flavors of particles: non-interacting Majorana spinons and localized fluxes ${ }^{5}$. Such excitations are long-lived, but belong to nontrivial topological sectors, and therefore cannot be created individually by any local operations. For the Kitaev spin liquid, the predicted continuum therefore represents coherent multiparticle excitations.

In contrast, upon moving away from the pure Kitaev point, the relevant symmetries that protect the spinons and fluxes are lifted both by additional magnetic interactions and by spontaneous symmetry breaking of the magnetic order. This tends to confine spinons into gauge neutral objects such as magnons ${ }^{47,48}$. Despite this latter tendency, we have argued that coherent magnons are unlikely to appear at large $\Gamma_{1}$ due to the strong anharmonicity in the magnon Hamiltonian. While this leaves open the possibility that nearly free Majorana spinons persist into the zigzag ordered phase in some regions of the Brillouin zone, a more general scenario is that the observed continua represent fully incoherent excitations. In this second scenario, the excitations are not describable in terms of any type of free particles with small decay rates and well-defined dispersions. The broad continua therefore reflect the absence of coherent quasiparticles altogether, rather than particular experimental selection rules related to fractionalization. At present, it is not clear which of these scenarios applies to the iridates and $\alpha-\mathrm{RuCl}_{3}$, although a key role must be played by both the Kitaev $K_{1}$ and off-diagonal couplings such as $\Gamma_{1}$. In any case, the study of these materials calls into question the stability of magnetic quasiparticles in the presence of strongly anisotropic interactions.

In summary, we have shown that all main features of the magnetic excitations in $\alpha-\mathrm{RuCl}_{3}{ }^{12}, 13,24$ are consistent with strongly anisotropic interactions having signs and relative magnitudes in agreement with ab initio predictions. The ferromagnetic Kitaev coupling $\left(K_{1}<0\right)$ is supplemented by a significant off-diagonal term $\left(\Gamma_{1}>0\right)$ that plays a crucial role in establishing both the zigzag order and the observed continua. In the presence of such interactions, the conventional magnon description breaks down even deep in the ordered phase, due to strong coupling of the one-magnon and two-magnon states. This effect is expected to persist over a large range of the phase diagram suggesting that the observed continua in $\alpha-\mathrm{RuCl}_{3}$ and the iridates $A_{2} \mathrm{IrO}_{3}$ represent a rich and general phenomenon extending beyond the Kitaev spin liquid. For this class of strongly spin-orbital-coupled magnets, the presence of complex and frustrated anisotropic interactions leads naturally to dominant anharmonic effects in the inelastic magnetic response. Fully describing the dynamics of these and similar materials therefore represents a formidable challenge that is likely to reveal aspects not found in conventional isotropic magnets.

\section{Methods}

Exact diagonalization. The neutron scattering intensity was computed via:

$$
\mathcal{I}(\mathbf{k}, \omega) \propto f^{2}(\mathbf{k}) \int d t \sum_{\mu, \nu}\left(\delta_{\mu, \nu}-k_{\mu} k_{\nu} / k^{2}\right) \times \sum_{i, j}\left\langle S_{i}^{\mu}(t) S_{j}^{\nu}(0)\right\rangle e^{-i \mathbf{k} \cdot\left(\mathbf{r}_{i}-\mathbf{r}_{j}\right)-i \omega t}
$$

where $f(\mathbf{k})$ is the atomic form factor of $\mathrm{Ru}^{3+}$ from ref. ${ }^{49}$. ED calculations were performed using the Lanczos algorithm ${ }^{50}$, on several 20 -site and 24-site clusters with periodic boundary conditions. Such periodic clusters are detailed in Supplementary Note 4 . Excitations were computed using the continued fraction method $^{51}$. Further details and additional results are presented in the Supplementary Notes 4 and 5; these extensive calculations go beyond previous ED studies $^{16,17,20,26,29}$, which focused mainly on the static properties, or a limited portion of the phase diagram. ED results shown for the high-symmetry $\Gamma, \mathrm{M}, \mathrm{Y}, \mathrm{X}$ and $\Gamma^{\prime}$ points were averaged over all clusters. The ED k-dependence of $\mathcal{I}(\mathbf{k}, \omega)$, integrated over the energy windows $E=1.3-2.3,5.5-8.5$, and $10.5+\mathrm{meV}$ (Figs. $2 \mathrm{c}$ $3 \mathrm{c})$, was obtained from a single 24-site cluster respecting all symmetries of the model. The discrete ED spectra were Gaussian broadened by $0.5 \mathrm{meV}$, consistent with the width of experimental features ${ }^{12}$. The intensities were also averaged over the same range of out-of-plane momentum as in the experiment ${ }^{12}$.

Linear spin-wave theory. LSWT results shown in Figs. 1 and 2 were obtained with the aid of SpinW ${ }^{52}$. Following the approach with the ED data, the discrete LSWT spectra were as well Gaussian broadened by $0.5 \mathrm{meV}$ and the intensities were also averaged over the same range of out-of-plane momentum as with ED and in the experiment $^{12}$

Imaginary self-consistent Dyson equation approach. In order to calculate magnon decay rates $\gamma_{\mathbf{k}, n}$, we have evaluated three-magnon interaction vertices by performing rotation to local reference frames of spins. The obtained value of the real-space interaction is quite large, about $\sim 3 \mathrm{meV}$. Next, the Born approximation calculation of the decay rates results in unphysical divergencies ${ }^{34}$, thus the selfenergy $\Sigma_{\mathbf{k}, n}$ needs to be regularized. We have used the so-called iDE approach: a 
self-consistent solution on the imaginary part of the Dyson's equation, $\Sigma_{\mathbf{k}, n}\left(\epsilon_{\mathbf{k}, n}+i \gamma_{\mathbf{k}, n}\right)=-i \gamma_{\mathbf{k}, n}$, see ref. ${ }^{40}$. We have obtained the regularized broadening for the magnon spectrum and have calculated the transverse part of the dynamical structure factor, shown in Fig. 6, by adding the calculated decay rates to experimental resolution of $0.25 \mathrm{meV}$. The spectral function is approximated as a Lorentzian. More technical details can be found in the Supplementary Note 3.

Code availabilty. Custom computer codes used in this study are available from the corresponding author upon reasonable request. Documentation of the codes is not available.

Data availability. Data are available from the corresponding author upon reasonable request.

Received: 31 March 2017 Accepted: 24 August 2017

Published online: 27 October 2017

\section{References}

1. Lifshitz, E. M. \& Pitaevskii, L. P. Statistical Physics Part 2 (Pergamon Press, Oxford, 1980).

2. Balents, L. Spin liquids in frustrated magnets. Nature 464, 199-208 (2010)

3. Coldea, R., Tennant, D. A., Tsvelik, A. M. \& Tylczynski, Z. Experimental realization of a 2D fractional quantum spin liquid. Phys. Rev. Lett. 86, 1335-1338 (2001).

4. Han, T.-H. et al. Fractionalized excitations in the spin-liquid state of a kagomelattice antiferromagnet. Nature 492, 406-410 (2012).

5. Kitaev, A. Anyons in an exactly solved model and beyond. Ann. Phys. 321, 2-111 (2006).

6. Jackeli, G. \& Khaliullin, G. Mott insulators in the strong spin-orbit coupling limit: from Heisenberg to a quantum compass and Kitaev models. Phys. Rev. Lett. 102, 017205 (2009).

7. Rau, J. G., Lee, E. K.-H. \& Kee, H.-Y. Spin-orbit physics giving rise to novel phases in correlated systems: iridates and related materials. Annu. Rev. Condens. Matter Phys. 7, 195-221 (2016).

8. Gretarsson, $\mathrm{H}$. et al. Magnetic excitation spectrum of $\mathrm{Na}_{2} \mathrm{IrO}_{3}$ probed with resonant inelastic X-ray scattering. Phys. Rev. B 87, 220407 (2013).

9. Hwan Chun, S. et al. Direct evidence for dominant bond-directional interactions in a honeycomb lattice iridate $\mathrm{Na}_{2} \mathrm{IrO}_{3}$. Nat. Phys. 11, 462-466 (2015).

10. Sandilands, L. J., Tian, Y., Plumb, K. W., Kim, Y.-J. \& Burch, K. S. Scattering continuum and possible fractionalized excitations in $\alpha-\mathrm{RuCl}_{3}$. Phys. Rev. Lett. 114, 147201 (2015).

11. Nasu, J., Knolle, J., Kovrizhin, D. L., Motome, Y. \& Moessner, R. Fermionic response from fractionalization in an insulating two-dimensional magnet. Nat. Phys. 12, 912-915 (2016).

12. Banerjee, A. et al. Neutron scattering in the proximate quantum spin liquid $\alpha$ - $\mathrm{RuCl}_{3}$. Science 356, 1055-1059 (2017).

13. Banerjee, A. et al. Proximate Kitaev quantum spin liquid behaviour in a honeycomb magnet. Nat. Mater. 15, 733-740 (2016).

14. Do, S.-H. et al. Incarnation of Majorana fermions in Kitaev quantum spin lattice. Preprint at https://arxiv.org/abs/1703.01081 (2017).

15. Glamazda, A., Lemmens, P., Do, S.-H., Choi, Y. S. \& Choi, K.-Y. Raman spectroscopic signature of fractionalized excitations in the harmonichoneycomb iridates $\beta$ - and $\gamma$ - $\mathrm{Li}_{2} \mathrm{IrO}_{3}$. Nat. Commun. 7, 12286 (2016).

16. Chaloupka, J., Jackeli, G. \& Khaliullin, G. Kitaev-Heisenberg model on a honeycomb lattice: possible exotic phases in iridium oxides $A_{2} \mathrm{IrO}_{3}$. Phys. Rev. Lett. 105, 027204 (2010)

17. Rau, J. G., Lee, E. K.-H. \& Kee, H.-Y. Generic spin model for the honeycomb iridates beyond the Kitaev limit. Phys. Rev. Lett. 112, 077204 (2014).

18. Winter, S. M., Li, Y., Jeschke, H. O. \& Valent, R. Challenges in design of Kitaev materials: magnetic interactions from competing energy scales. Phys. Rev. B 93, 214431 (2016)

19. Sears, J. A. et al. Magnetic order in $\alpha-\mathrm{RuCl}_{3}$ : a honeycomb-lattice quantum magnet with strong spin-orbit coupling. Phys. Rev. B 91, 144420 (2015).

20. Chaloupka, J. \& Khaliullin, G. Magnetic anisotropy in the Kitaev model systems $\mathrm{Na}_{2} \mathrm{IrO}_{3}$ and $\mathrm{RuCl}_{3}$. Phys. Rev. B 94, 064435 (2016).

21. Kimchi, I., Coldea, R. \& Vishwanath, A. Unified theory of spiral magnetism in the harmonic-honeycomb iridates $\alpha, \beta$, and $\gamma \mathrm{Li}_{2} \mathrm{IrO}_{3}$. Phys. Rev. B 91, 245134 (2015).

22. Kim, H.-S., Lee, E. K.-H. \& Kim, Y.-B. Predominance of the Kitaev interaction in a three-dimensional honeycomb iridate: from ab initio to spin model. EPL 112, 67004 (2015)

23. Cao, H. B. et al. Low-temperature crystal and magnetic structure of $\alpha-\mathrm{RuCl}_{3}$. Phys. Rev. B 93, 134423 (2016).

24. Ran, K. et al. Spin-wave excitations evidencing the Kitaev interaction in single crystalline $\alpha-\mathrm{RuCl}_{3}$. Phys. Rev. Lett. 118, 107203 (2017).

25. Kim, H.-S. \& Kee, H.-Y. Crystal structure and magnetism in $\alpha-\mathrm{RuCl}_{3}$ : an $a b$ initio study. Phys. Rev. B 93, 155143 (2016).
26. Yadav, R. et al. Kitaev exchange and field-induced quantum spin-liquid states in honeycomb $\alpha$-RuCl 3 . Sci. Rep. 6, 37925 (2016).

27. Wang, W., Dong, Z.-Y., Yu, S.-L. \& Li, J.-X. Theoretical investigation of the magnetic dynamics and superconducting pairing symmetry in $\alpha-\mathrm{RuCl}_{3}$. Phys . Rev. B 96, 115103 (2017).

28. Hou, Y. S., Xiang, H. J. \& Gong, X. G. Unveiling magnetic interactions of ruthenium trichloride via constraining direction of orbital moments: potential routes to realize a quantum spin liquid. Phys. Rev. B 96, 054410 (2017).

29. Katukuri, V. M. et al. Kitaev interactions between $j=1 / 2$ moments in honeycomb $\mathrm{Na}_{2} \mathrm{IrO}_{3}$ are large and ferromagnetic: insights from ab initio quantum chemistry calculations. New J. Phys. 16, 013056 (2014).

30. Gohlke, M., Verresen, R., Moessner, R. \& Pollmann, F. Dynamics of the KitaevHeisenberg model. Preprint at https://arxiv.org/abs/1701.04678 (2017).

31. Johnson, R. D. et al. Monoclinic crystal structure of $\alpha-\mathrm{RuCl}_{3}$ and the zigzag antiferromagnetic ground state. Phys. Rev. B 92, 235119 (2015).

32. Harris, A. B., Kumar, D., Halperin, B. I. \& Hohenberg, P. C. Dynamics of an antiferromagnet at low temperatures: spin-wave damping and hydrodynamics Phys. Rev. B 3, 961-1024 (1971).

33. Chernyshev, A. L. \& Zhitomirsky, M. E. Magnon decay in noncollinear quantum antiferromagnets. Phys. Rev. Lett. 97, 207202 (2006).

34. Zhitomirsky, M. E. \& Chernyshev, A. L. Colloquium: spontaneous magnon decays. Rev. Mod. Phys. 85, 219-242 (2013).

35. Ruvalds, J. \& Zawadowski, A. Two-phonon resonances and hybridization of the resonance with single-phonon states. Phys. Rev. B 2, 1172-1175 (1970).

36. Kosevich, A. M. The Crystal Lattice: Phonons, Solitons, Dislocations, Superlattices (Wiley-VCH Verlag GmbH \& Co. KGaA, Weinheim, 2005).

37. Sizyuk, Y., Wölfle, P. \& Perkins, N. B. Selection of direction of the ordered moments in $\mathrm{Na}_{2} \mathrm{IrO}_{3}$ and $\alpha-\mathrm{RuCl}_{3}$. Phys. Rev. B 94, 085109 (2016).

38. Chaloupka, J. \& Khaliullin, G. Hidden symmetries of the extended KitaevHeisenberg model: implications for the honeycomb-lattice iridates $A_{2} \mathrm{IrO}_{3}$. Phys. Rev. B 92, 024413 (2015).

39. Hwang, K. \& Kim, Y. B. Theory of triplon dynamics in the quantum magnet $\mathrm{BiCu}_{2} \mathrm{PO}_{6}$. Phys. Rev. B 93, 235130 (2016)

40. Maksimov, P. A., Zhitomirsky, M. E. \& Chernyshev, A. L. Field-induced decays in XXZ triangular-lattice antiferromagnets. Phys. Rev. B 94, 140407 (2016).

41. Mourigal, M., Fuhrman, W. T., Chernyshev, A. L. \& Zhitomirsky, M. E. Dynamical structure factor of the triangular-lattice antiferromagnet. Phys. Rev. B 88, 094407 (2013).

42. Biffin, A. et al. Noncoplanar and counterrotating incommensurate magnetic order stabilized by Kitaev interactions in $\gamma-\mathrm{Li}_{2} \mathrm{IrO}_{3}$. Phys. Rev. Lett. 113, 197201 (2014).

43. Biffin, A. et al. Unconventional magnetic order on the hyperhoneycomb Kitaev lattice in $\beta-\mathrm{Li}_{2} \mathrm{IrO}_{3}$ : full solution via magnetic resonant X-ray diffraction. Phys. Rev. B 90, 205116 (2014).

44. Williams, S. C. et al. Incommensurate counterrotating magnetic order stabilized by Kitaev interactions in the layered honeycomb $\alpha-\mathrm{Li}_{2} \mathrm{IrO}_{3}$. Phys. Rev. B 93, 195158 (2016).

45. Takayama, T. et al. Hyperhoneycomb iridate $\beta-\mathrm{Li}_{2} \mathrm{IrO}_{3}$ as a platform for Kitaev magnetism. Phys. Rev. Lett. 114, 077202 (2015).

46. Kim, H.-S., Kim, Y. B. \& Kee, H.-Y. Revealing frustrated local moment model for pressurized hyperhoneycomb iridate: paving the way toward a quantum spin liquid. Phys. Rev. B 94, 245127 (2016).

47. Mandal, S., Bhattacharjee, S., Sengupta, K., Shankar, R. \& Baskaran, G. Confinement-deconfinement transition and spin correlations in a generalized Kitaev model. Phys. Rev. B 84, 155121 (2011).

48. Schaffer, R., Bhattacharjee, S. \& Kim, Y.-B. Quantum phase transition in Heisenberg-Kitaev model. Phys. Rev. B 86, 224417 (2012).

49. Cromer, D. T. \& Waber, J. T. Scattering factors computed from relativistic Dirac-Slater wave functions. Acta Cryst. 18, 104-109 (1965).

50. Lanczos, C. An iteration method for the solution of the eigenvalue problem of linear differential and integral operators. J. Res. Nat. Bur. Stand. 45, 255-282 (1950).

51. Dagotto, E. Correlated electrons in high-temperature superconductors. Rev. Mod. Phys. 66, 763-840 (1994).

52. Toth, S. \& Lake, B. Linear spin-wave theory for single-Q incommensurate magnetic structures. J. Phys. Condens. Matter 27, 166002 (2015).

53. Knolle, J., Kovrizhin, D. L., Chalker, J. T. \& Moessner, R. Dynamics of fractionalization in quantum spin liquids. Phys. Rev. B 92, 115127 (2015).

54. Knolle, J., Kovrizhin, D. L., Chalker, J. T. \& Moessner, R. Dynamics of a twodimensional quantum spin liquid: signatures of emergent Majorana fermions and fluxes. Phys. Rev. Lett. 112, 207203 (2014).

\section{Acknowledgements}

The authors acknowledge useful discussions with J. Chaloupka, A. Banerjee, S.E. Nagler A.A. Tsirlin, R. Moessner, F. Pollmann, and M. Zhitomirsky. S.M.W. acknowledges support through an NSERC Canada Postdoctoral Fellowship. R.V. and K.R. acknowledge support by the Deutsche Forschungsgemeinschaft through grant SFB/TR 49. The work of P.A.M. and A.L.C. was supported by the U.S. Department of Energy, Office of Science, Basic Energy Sciences under award no. DE-FG02-04ER46174 


\section{Author contributions}

R.V. and S.M.W. conceived the project. K.R., S.M.W. and A.H. performed and analyzed the ED calculations. P.A.M. and A.L.C. performed and analyzed the iDE results. All authors contributed equally to the manuscript.

\section{Additional information}

Supplementary Information accompanies this paper at doi:10.1038/s41467-017-01177-0.

Competing interests: The authors declare no competing financial interests.

Reprints and permission information is available online at http://npg.nature.com/ reprintsandpermissions/

Publisher's note: Springer Nature remains neutral with regard to jurisdictional claims in published maps and institutional affiliations. (c) (i) Open Access This article is licensed under a Creative Commons Attribution 4.0 International License, which permits use, sharing, adaptation, distribution and reproduction in any medium or format, as long as you give appropriate credit to the original author(s) and the source, provide a link to the Creative Commons license, and indicate if changes were made. The images or other third party material in this article are included in the article's Creative Commons license, unless indicated otherwise in a credit line to the material. If material is not included in the article's Creative Commons license and your intended use is not permitted by statutory regulation or exceeds the permitted use, you will need to obtain permission directly from the copyright holder. To view a copy of this license, visit http://creativecommons.org/ licenses/by/4.0/

(C) The Author(s) 2017 